\title{
FLUTUAÇÃO POPULACIONAL DE Whitius piger (PSEUDOSCORPIONIDA) EM GRANJA AVÍCOLA EM PELOTAS, RS, BRASIL
}

\author{
(Population flotation of Whitius piger (Pseudoscorpionida) in poultry farm in Pelotas, RS, Brazil)
}

PINTO, D.M..'; RIBEIRO, P.B. ${ }^{2}$; SILVEIRA JR., P. ${ }^{3}$; BERNARDI, E. ${ }^{4}$

\begin{abstract}
1'Departamento de Microbiologia e Parasitologia, Universidade Federal de Pelotas, Caixa Postal 354, CEP96010-900, Pelotas-RS/ dimoscarelli@yahoo.com.br; ${ }^{2}$ Departamento de Microbiologia e Parasitologia, Universidade Federal de Pelotas, Pelotas-RS; ${ }^{3}$ Instituto de Física e Matemática, Universidade Federal de Pelotas, Pelotas-RS; ${ }^{4}$ Departamento de Microbiologia e Parasitologia, Universidade Federal de Pelotas, Pelotas-RS.
\end{abstract}

RESUMO - Para avaliar a flutuação populacional de Whitius piger foram utilizados dois métodos de monitoramento, armadilha do tipo "tubo" e do tipo "sanduíche". O experimento realizou-se no período de abril de 2002 a março de 2003, em aviário do Conjunto Agrotécnico Visconde da Graça (CAVG), localizado em Pelotas, Rio Grande do Sul, Brasil. Nesse período foram capturados 1539 exemplares de Whitius piger, sendo o período de maior ocorrência no mês de março (312), quando a temperatura média mensal foi de $22,2^{\circ} \mathrm{C}$ e a menor ocorrência foi no mês de junho (11) com temperatura média mensal de 14,5ㅇ. O pseudoescorpionideo esteve presente nos 12 meses de coleta, apresentando maior captura nos meses de maior temperatura e menor captura nos meses de menor temperatura.

Palavras-chave: Whitius piger, flutuação populacional, aviário, Pseudoscorpionida.

ABSTRACT - To evaluate the populational flotation of Whitius piger two monitoring methods were used, a tube type trap and a sandwich type trap. The experiment took place in the period of April 2002 to March of 2003, in an aviary from the Conjunto Agrotécnico Visconde da Graça (CAVG), located at Pelotas, Rio Grande do Sul, Brazil. During that period 1539 samples of Whitius piger were captured, being the period of larger occurrence in the month of March (312), when the monthly medium temperature was $22,2^{\circ} \mathrm{C}$ and the smallest occurrence during the month of June (11), with a monthly medium temperature of $14,5^{\circ} \mathrm{C}$. It has been found that the Whitius piger was present iduring all the 12 months of collection, displaying a larger capture during the months of higher temperatures and a smaller capture in the months of lower temperatures.

Key-words: Whitius piger, population flotation, aviary, Pseudoscorpionida.

\section{Introdução}

A necessidade de produzir quantidades cada vez maiores de alimentos levou o homem a procurar formas de produção intensivas. Com esse propósito, uma das alternativas para maior produção de alimentos de origem animal foi a criação intensiva através de confinamento. Tal medida aumentou a concentração de excretas de animais, criando, nestes locais, ecossistemas artificiais propícios à proliferação de artrópodes. Dessa forma algumas espécies de artrópodes passaram a viver sinantropicamente e por algumas delas serem vetores de patógenos, apresentam grande importância médica e veterinária (FRANCISCO, 1996).

A diversidade de artrópodes encontrada em esterco acumulado, nos locais de criação de aves domésticas, é muito grande, e esses artrópodes são principalmente, espécies de coleópteros, dípteros ciclorrafos e ácaros (AXTELL e ARENDS, 1990).

$\mathrm{O}$ estudo das espécies que ocorrem em ambientes modificados pelo homem, assume importância não só ecológica, pois a 
associação destas espécies à veiculação dos mais diversos organismos patogênicos, se reveste de interesse sanitário (MASCARINI, 1995).

Whitius piger teve sua ocorrência em aviários relatada pela primeira vez, por $\mathrm{BICHO}$ (2001), em fezes com diferentes estágios de decomposição.

W. pigeré predador de Dermanyssus gallinae e segundo MUCHMORE (1992), os pseudoescorpiões alimentam-se de líquidos corpóreos de outros artrópodes e pequenos invertebrados. PECK e ANDERSON (1969) referem-se aos Pseudoscorpionida com os predadores de formas jovens de dípteros.

O presente trabalho tem como objetivo avaliar a ocorrência deste pseodoscorpionida, através de diferentes tipos de armadilhas, visando conhecer a freqüência deste predador ao longo do ano para poder utilizar este conhecimento em futuros programas de controle biológico.

\section{Método}

O trabalho foi realizado na granja do Conjunto Agrotécnico Visconde da Graça (CAVG), pertencente a Universidade Federal de Pelotas, em Pelotas, RS, no período de abril de 2002 a março de 2003.

A coleta foi realizada através de dois métodos, com o objetivo de verificar freqüência deste artrópodo ao longo do ano.

Os métodos de coleta utilizados foram:

- Armadilha do tipo "tubo" ou de Arends (SAFRIT e AXTELL, 1984) - consiste em um tubo de polivinilcloreto (PVC) de $3,8 \mathrm{~cm}$ de diâmetro por $23 \mathrm{~cm}$ de comprimento, contendo em seu interior papel corrugado, colocado de forma que as ondulações fiquem dispostas em sentido longitudinal ao tubo.

- Armadilha do tipo "sanduíche" (SAFRIT e AXTELL, 1984) - consiste em caixas de madeira de $20 \mathrm{~cm}$ de comprimento por $15 \mathrm{~cm}$ de largura e $8 \mathrm{~cm}$ de altura, com tampa e duas aberturas de $1 \mathrm{~cm}$ no sentido longitudinal, junto a base da caixa, para facilitar a entrada do coleóptero. No interior das armadilhas foi colocado papel corrugado, de modo que preenchesse a caixa.
Foram instaladas 16 armadilhas, em grupos de quatro, em quatro locais diferentes do galpão. Cada grupo estava composto por duas armadilhas do tipo "sanduíche" e duas armadilhas do tipo "tubo". As armadilhas foram dispostas no piso do aviário, sobre a cama (maravalha), dispostas lado a lado equidistantes $30 \mathrm{~cm}$ umas das outras.

Foram feitas coletas semanais, onde o conteúdo de cada armadilha com o auxilio de um funil de alumínio era colocado em um pote plástico, devidamente identificado. Os papéis corrugados das armadilhas eram substituídos por papéis novos e os artrópodes capturados eram levados para o laboratório onde eram feitas triagens, identificação e contagem dos mesmos. O material encontrado, em cada armadilha, bem como a data e as observações pertinentes foram registrados em ficha controle. Após esse procedimento, os pseudoscorpionideos foram colocados em potes de vidro contendo álcool e devidamente identificados. Os dados de temperatura e precipitação pluviométrica foram fornecidos pela Estação Climatológica do conjunto Agrotécnico Visconde da Graça.

Os resultados obtidos foram submetidos a analise de regressão polinomial, utilizando-se o programa estatístico SANEST (ZONTA e MACHADO, 1984).

\section{Resultados e Discussão}

Durante o período experimental, foram capturados 45.764 artrópodes pertencentes às Classes Insecta e Arachnida. A Classe Insecta representou $96,64 \%$, do total de artrópodes capturados. Whitius piger foi o único aracnídeo capturado num total de 1536 espécimens (TABELA 1).

A flutuação populacional de Withius piger capturada em galpão de poedeiras, pode ser observada na FIGURA 2.

O modelo ajustado foi o quadrático, com significância a 0,009\%, com coeficiente de determinação de $76,43 \%$.

O modelo ajustado foi: $y_{i}=2,1150-0,2105 x_{i}$ $+0,0333 x_{i}^{2}$, onde $i=1,2, \ldots, 11,12$ (ordem dos meses do ano). 
FIGURA 1 - TEMPERATURA MÉDIA MENSAL E PRECIPITAÇÃO PLUVIOMÉTRICA, NO PERÍODO DE ABRIL DE 2002 A MARÇO DE 2003, EM PELOTAS - RS.

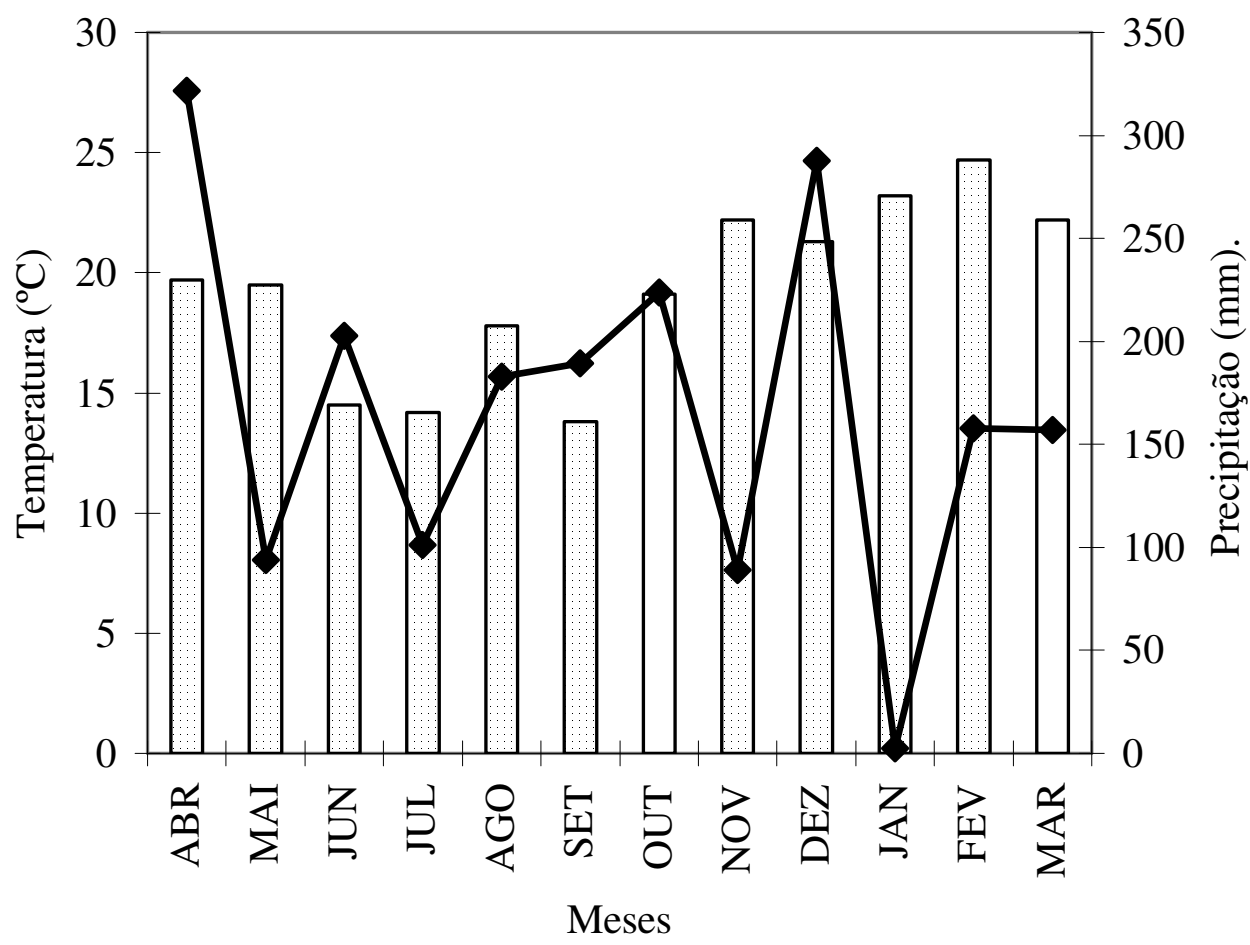

$\square$ Temperatura —-Preciptação Pluviométrica

FIGURA 2 - REGRESSÃO POLINOMIAL DA FLUTUAÇÃO POPULACIONAL DE Withius piger, EM GRANJA AVÍCOLA, NO PERÍODO DE ABRIL DE 2002 A MARÇO DE 2003, EM PELOTAS - RS.

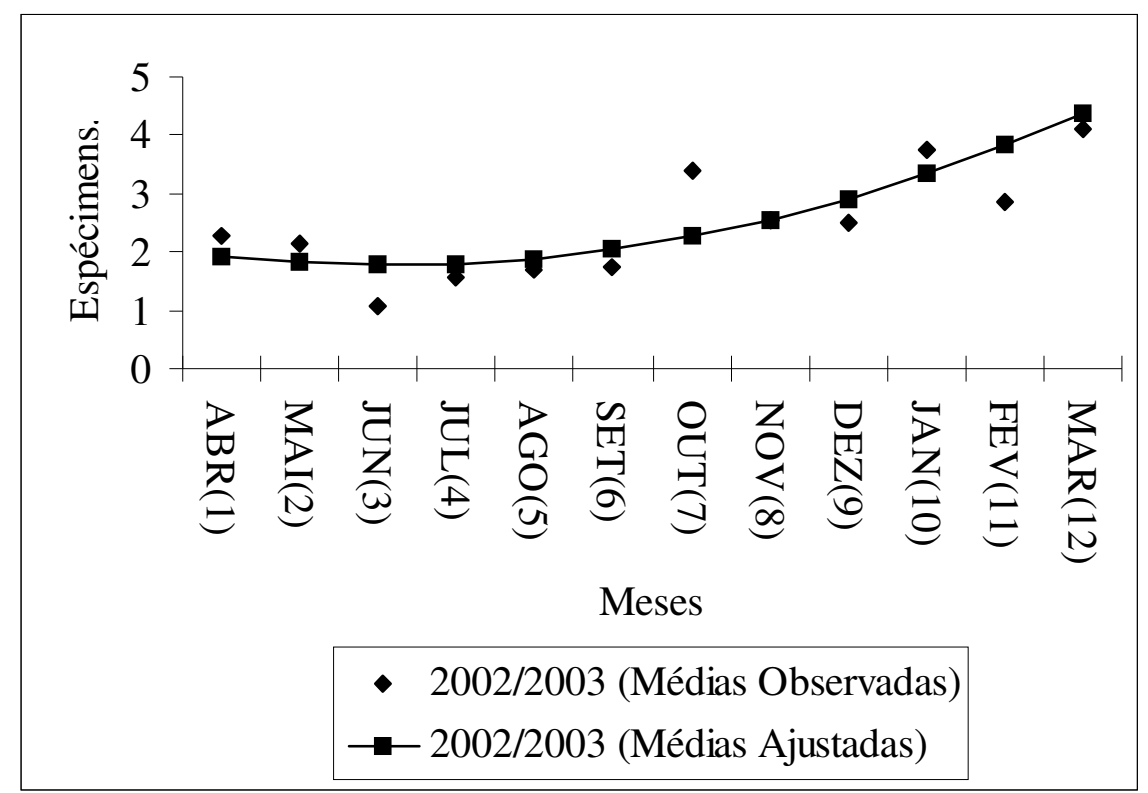


Os resultados encontrados na avaliação da flutuação populacional do pseudoescorpionideo Withius piger, estão de acordo com os resultados obtidos por $\mathrm{BICHO}$ (2001), que registrou pela primeira vez a presença deste aracnídeo em aviários no Brasil, durante todo o ano, através da avaliação de fezes em vários períodos de decomposição.

A maior freqüência do pseudoscorpionideo nos meses de maior temperatura, esta de acordo com ODUM (1988), que se refere aos fatores extrínsicos e intrínsicos, como sendo controladores das flutuações anuais. Esses fatores, principalmente a temperatura, explicam as variações da flutuação populacional que foram observadas durante os 12 meses avaliados demonstrando, portanto, que as exigências bióticas e abióticas são próprias da espécie.

\section{Conclusão}

Withius piger ocorreu durante todos os meses do ano, variando a densidade populacional ao longo do mesmo, sendo as maiores médias de captura obtidas nos meses com maiores temperaturas médias.

A precipitação pluviométrica não influenciou a população do pseudoescorpionideo.

\section{REFERÊNCIAS}

AXTELL, R.C.; ARENDS, J.J. Ecology and management of arthropod pests of poultry. Annual Review of Entomology, Palo Alto, n. 35, p.101-126, 1990.

Recebido para publicação: 04/07/2005

Aprovado:

$30 / 10 / 2005$
BICHO, C.L. Comunidade de artrópodes e flutuação populacional circanual de dípteros e coleópteros, em granja avícola, em Pelotas - RS. Curitiba, 2001. 110 p. Tese (Doutorado) Universidade Federal do Paraná.

FRANCISCO, O. Alphitobius diaperinus (Panzer) (Coleoptera: Tenebrionidae) associado a esterco em granjas de aves poedeiras: fenologia, estrutura etária e parasitismo. Campinas, 1996. 116 p. Dissertação (Mestrado) - Universidade Estadual de Campinas.

MASCARINI, L.M. Aspectos biológicos de Muscina stabulans (Fallén, 1817) em condições de laboratório. Campinas, 1995. 68 p. Dissertação (Mestrado) - Universidade de Campinas.

MUCHMORE, W.B. Pseudoscorpionida. In: SYNOPSIS and classification of living organisms. New York: McGraw Hill Book , 1992. p. 96-102.

ODUM, E. Ecologia. Rio de Janeiro: Guanabara Koogan, 1988. 434 p.

PECK, J.H.; ANDERSON, J.R. Arthropod predators of immature Díptera developing in poultry dropping in Northern Califórnia Part I. Determination of seasonal abundance and natural cohabitation with prey. Journal of Medical Entomology, Lanham, n. 6, p. 163-167, 1969.

SAFRIT, R.D.; AXTELL R.C. Evaluations of sampling methods for darkling beetles (Alphitobius diaperinus) in the litter of turkey and broiler houses. Poultry Science, v. 63, n. 12, p. 2368-2375, 1984.

ZONTA, E.P.; MACHADO, A.A. SANEST - Sistema de Análise Estatística para Microcomputadores. Registrado na Secretaria Especial de Informática sob ํ‥066060 - categoria A. Pelotas: Universidade Federal de Pelotas, 1984. 